\title{
OTA Testing for Massive MIMO Devices Using Cascaded APM Networks and Channel Emulators
}

\author{
Lijian Xin $\mathbb{D}^{1},{ }^{1}$ Yong Li $\mathbb{D},{ }^{1}$ Hao Sun $\mathbb{D}^{1},{ }^{1}$ and Xiang Zhang $\mathbb{D}^{2}$ \\ ${ }^{1}$ Key Laboratory of Universal Wireless Communications (Ministry of Education), \\ Beijing University of Posts and Telecommunications, Beijing 100876, China \\ ${ }^{2}$ China Academy of Information and Communications Technology, Beijing 100191, China \\ Correspondence should be addressed to Xiang Zhang; zhangxiang1@caict.ac.cn
}

Received 6 May 2019; Revised 17 July 2019; Accepted 13 September 2019; Published 12 November 2019

Guest Editor: Danping He

Copyright ( 2019 Lijian Xin et al. This is an open access article distributed under the Creative Commons Attribution License, which permits unrestricted use, distribution, and reproduction in any medium, provided the original work is properly cited.

\begin{abstract}
This paper proposes an over-the-air (OTA) testing setup for millimeter-wave (mmWave) massive multiple-input multiple-output (MIMO) equipment using cascaded amplitude and phase modulation (APM) network and channel emulator. Compared with the existing test setup with mechanical switch, the proposed testing setup enables more accurate reconstruction of the radio channel environment under the multiprobe anechoic chamber (MPAC) setup without increasing the number of channel emulators (CEs) to control the system cost. The constructed MPAC testing setup for mmWave and massive MIMO equipment is composed of an anechoic chamber, a sectored probe wall containing a number of probes, an APM network, a fading channel emulator, and a user emulator (UE). In this paper, the structural model and the performance advantages of the proposed radiated testing setup are described, and a fully connected APM network for radiated testing is more prominent than the existing switch. Moreover, the angular spectrum is selected as the performance metric for the reconstructed channel. The ability of the proposed system to reconstruct the power angular spectrum (PAS) of the target channel is studied under both static and dynamic channel models, which can reflect the performance of beamforming procedures of the massive MIMO antenna arrays, e.g., beam acquisition, tracking, and refinement. The simulation results for angular spectrum support the superiority of the proposed OTA testing setup. Furthermore, the simulations for average channel capacity also show that radiated testing setup using cascaded APM network and channel emulator is valid.
\end{abstract}

\section{Introduction}

Due to the large amount of available spectrum in the millimeter-wave (mmWave) band, mmWave communication has become an important technology in $5 \mathrm{G}$ cellular communication systems. Compared with sub-6 GHz communication, mmWave channel has higher penetration losses and energy losses due to scattering, thereby resulting in high sparseness and specular reflection of the channel [1]; thus, the signal is subject to higher pathloss and transmission congestion at mmWave bands. In order to overcome pathloss and maintain high signal power, the signal-to-interference-plus-noise ratio (SINR) should have a larger value, which means that mmWave antenna should have a higher gain. The antenna array can be used to improve the reliability of the communication link and compensate for severe pathloss to increase SINR [2]. Fortunately, the wavelength for the mmWave frequency is shorter, and the actual size of the mmWave antenna is smaller than the antenna size in sub-6 GHz. Therefore, massive multiple-input multiple-output (MIMO) antenna system can be configured with more antennas to increase signal power. It is well known that massive MIMO is one of the key technologies in $5 \mathrm{G}$ communication, and evaluating the performances of both antennas and baseband parts is significant for the design and development of mmWave massive MIMO devices. Given the evaluation demands of the mmWave equipment in the future, the test for controllable antenna systems is an unprecedented task. 
For sub- $6 \mathrm{GHz}$ antenna systems, conducted testing is used to test devices typically. However, there are two main reasons why the conducted testing is no longer suitable for the mmWave antenna system [3]. Firstly, the mmWave system with tens or hundreds of antennas is a highly integrated unit. Therefore, given the system cost, size, and design difficulties, it is no longer applicable to test mmWave antenna devices using radio frequency (RF) cable. Secondly, the beamforming capability of the base station (BS) should be taken into account for the mmWave test. It is expected that the best beam will be found at both ends of the link during the link establishment process and then aligned continuously under time-variant radio channel conditions. For example, when one or both ends of a link are moving, beam acquisition and beam alignment are key features for mmWave device test. However, conducted testing ignores the beam features of massive MIMO devices. Therefore, given the infeasibility of conducted testing, mmWave devices should be performed through radiated testing in which the radiated antennas are used as the interface directly.

Recently, for the mmWave massive MIMO antenna testing, a three-dimensional (3D) sectored multiprobe anechoic chamber (MPAC) setup is proposed [4]. The MPAC OTA testing setup for the massive MIMO equipment is composed of an anechoic chamber which can shield external interference signals and absorb reflected waves, a sectored probe wall which can place a large number of probes, a switch system (mechanical relay switch: the key parameters, e.g., switching speed, insertion loss, isolation, cost, crosstalk, and lifetime, are dependent on the manufacturing technology of the switch), a channel emulator, and an user emulator. The procedure of the massive MIMO OTA testing and the configuration of the sectored OTA setup are detailed in [5].

In order to test the beam acquisition and beam tracking of mmWave massive MIMO equipment, a dynamic channel environment needs to be reproduced [6]. The reasons are as follows. Firstly, the antenna array and beamformer are important parts of the mmWave devices, and the beamforming is indispensable in the link establishment process. Therefore, the test for the adaptive beamforming requires the dynamic radio channel urgently. Secondly, the link distance in a massive MIMO system may be short, and small motions may significantly change the channel propagation conditions, causing rapid changes of the power amplitude observed by different multipath components. As mentioned above, the mmWave dynamic channel needs to be reconstructed in the MPAC setup.

In the existing OTA testing systems, it is assumed that the switch configuration is completely free and can be switched at every considered moment without any distortion [6]. However, the dynamic environment has strict performance requirements for mechanical relay switches. The performance of the mechanical relay switch was tested for high-performance reconfigurable RF circuits in [7]. There are some major problems in using mechanical relay switches. Firstly, from low frequency to high frequency, the switch isolation is reduced, and the insertion loss of the device is increased significantly, which may increase the noise of the system directly and affect the performance of the relay switches at high frequencies. Secondly, during the switching of mechanical relay switches, any idle open transmission line can resonate in the microwave range, and the resonance can reflect electrical energy to a source of the operating RF, thereby damaging it. For systems operating at $26 \mathrm{GHz}$ or higher frequencies, the above damage will be more severe due to the greatly reduced isolation [7]. Thirdly, the service life of the mechanical switch is short, and its components are sensitive to vibration. Longer service life requires more robust actuators and drive linkages which require that magnetic efficiency and mechanical rigidity are optimized. Last but not least, the mechanical switch can only use a subset of probes on the probe wall at each channel time sample, which wastes hardware resources and could not improve the performance of the MPAC. Therefore, in order to test mmWave massive MIMO devices accurately, higher technical requirements are put forward for the design of switches. Given the shortcomings of the mechanical relay switch, a new system structure is required and applied to the mmWave massive MIMO OTA testing system.

In view of the above drawbacks of the current mmWave OTA testing system with switches, this paper proposes the amplitude and phase modulation (APM) network, which is fully connected and digital, to replace the mechanical switch in the MPAC setup. Therefore, the MPAC testing setup with a cascaded APM network and channel emulator (CE) model is presented in this paper. The inputs of the APM network are connected to the outputs of the $\mathrm{CE}$, and the outputs of the APM network are connected to the probes. The main advantages of the OTA testing setup with the cascaded model are as follows:

(i) Firstly, given the reduced channel isolation of the switch from low to high frequencies, the channels of the fully connected APM network are independent of each other. In addition, there is no distortion of the mechanical relay switch mentioned above when reconstructing the dynamic channel using digital APM network. In addition, the update speed of the APM network can be synchronized with the sampling rate of the channel emulator. Furthermore, the APM network has an automatic verification function to balance the amplitude and phase deviation caused by different RF lines.

(ii) Secondly, the digital APM network can use all the probes placed on the probe wall at each sampling time of the dynamic channel, rather than a subset of the probes as in the case of the switch, thereby making full use of hardware resources and improving the accuracy of the emulated channel. Therefore, using the OTA testing setup with a cascaded model, the channel reconstruction capability can be improved greatly without increasing the number of channel emulators in the MPAC setup.

However, nothing is perfect. Potential practical challenges of the APM network will be discussed in the summary of simulation results, such as resolution of phase shifters 
(=word length), step size, and accuracy of analogue attenuators. In the future work, we will examine the influence of the above nonideal factors on channel reconstruction.

From the above descriptions, it is not difficult to find that the APM network's structure is simpler than that of the channel emulator and can only modulate the amplitude and phase of the radio frequency signals. In addition, considering a digital real-time control for APM structure, the APM network is the same as the channel emulator when generating impulse response of a single cluster. However, given the multiple clusters for a geometry-based stochastic channel model (GSCM), the APM network is powerless due to its simple structure. Therefore, the channel emulator cannot be fully replaced by the APM network, and the cost of simple APM network is lower than that of complex channel emulator. When reconstructing GSCM (multiple clusters) or generating mmWave channel, we need to add number of channel emulators to reconstruct the spatial characteristics of the target channel more accurately. Despite the APM network performance advantages described above, it is also expected to improve the accuracy of the reconstructed channel and greatly reduce test costs. Moreover, APM network is very convenient to adjust the amplitude and phase by loading data files which can be obtained in the sequel.

The notation used in this paper is as follows. Section 2 presents the structure of the APM network and the OTA testing system with a cascaded model. Section 3 details the MPAC setup with APM network for massive MIMO devices, and specific implementation process is analysed and summarized. The angular spectrum and the average channel capacity are selected as the performance metrics for the reconstructed channel. Numerical results and simulation comparisons are given in Section 4. Finally, conclusions are drawn in Section 5.

\section{OTA Testing Setup Model}

In the MPAC OTA testing setup, the relationship between the geometric test area and the accuracy of the reconstructed radio channel is a key issue. Using prefaded signals synthesis (PFS) technology, the purpose is to select proper probes and configure the optimal weights to reconstruct the spatial characteristics in the test area [8], so that the target PAS and the discrete PAS are as similar as possible. In the OTA testing for massive MIMO devices, the device under test (DUT) usually has larger antenna radius and higher beam resolution. Thus, more active probes are needed to recreate the target channel. The relation between the number of probes and the radius of the test area is given in [9], which indicates that increasing the number of probes can reconstruct the target channel more accurately within a given test area. Traditionally, each probe is connected to an output of the channel emulator, and adding probes means more channel emulator resources, which will greatly increase the overall cost of the OTA testing setup. Inspired by this, the paper proposes the MPAC testing setup using cascaded APM network and CE to achieve the same effect of the multiple channel emulators, improving the performance of the testing system and controlling the overall system cost.
2.1. Structure and Performance of the APM Network. Figure 1 shows physical map (output $A$ ports and input $B$ ports; the amplitude and the phase of each link can be updated) of the APM network. As shown in Figure 2, the fully connected structure of the APM network (Figure 2(a)) is very different from the mechanical switch (Figure 2(b)) which selects the output ports through switching at each time. Each output port connected to probe $A_{k}$, $k=1,2, \ldots, K$, and each input port connected to channel emulator $B_{p}, p=1,2, \ldots, P$, are cross-connected. That is, each output port of the APM network is superimposed by all input signals. The amplitude and the phase of each internal link can be updated at each sampling time and can be controlled by loading the amplitude and phase which can be set to either complex or real numbers.

Compared with mechanical switches, the main advantages of APM networks can be summarized as follows: Firstly, cross-connected links between the inputs and outputs are independent of each other, and their weights can be adjusted digitally. In addition, there is no resonance problem that may occur in the switching process of the switch. Secondly, amplitudes and phases of the fully connected links take effect within 1 millisecond quickly. The digital switching process only needs to change the amplitudes and phases of the RF channels, and there is no disturbance problem which has been described for the mechanical switching process of the switch. Thirdly, the switch and the additional RF lines may cause amplitude and phase errors of different probe links. However, the APM network has the ability of autocalibration to balance errors caused by the RF lines. Last but not least, clock calibration is another important function of the APM network. Due to the faster response time compared with the switch, the APM network can synchronize with the channel emulator, and the weights inside the APM network can be adjusted precisely in the reconstructing process of the dynamic channel.

\subsection{MPAC Setup with a Cascaded Model. Components of the} proposed MPAC OTA testing setup are shown in Figure 3. Unlike the testing setup of the 2D OTA ring structure, the DUT is placed at the edge of the anechoic chamber so that the space of the chamber can be utilized fully to control system costs. The DUT is placed in the center of the test area, and the distance from each point on the sectored probe wall to the test area center is equal. The fully connected APM network which can adjust the amplitude and phase of each internal link replaces the original switch device. In the uplink communication system, the $K$ outputs of the APM network are connected to $K$ probes, and $P$ inputs of the APM network are connected to $P$ outputs of the fading channel emulators $(K>P)$. The end of MPAC OTA testing setup is the user emulator.

It is worth noting that the mechanical switch uses a subset of all probes to emulate a static or dynamic channel. The number of probes used to synthesize the cluster is determined by the $P$ outputs of the CE. That is, $(K-P)$ probes are idle, and therefore, the probe resources are wasted. In addition, the APM network which adjusts the 


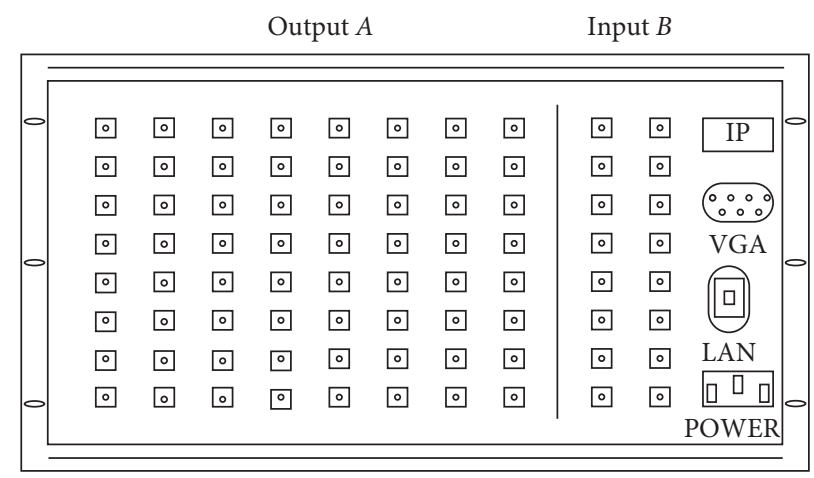

FIGURE 1: A physical map of the APM network equipment.

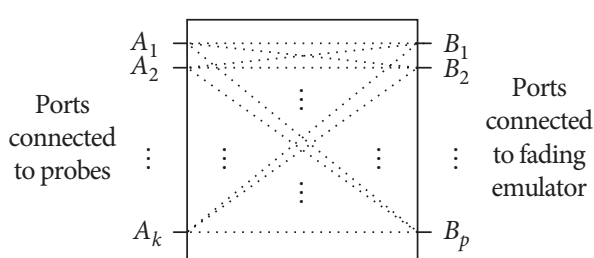

(a)

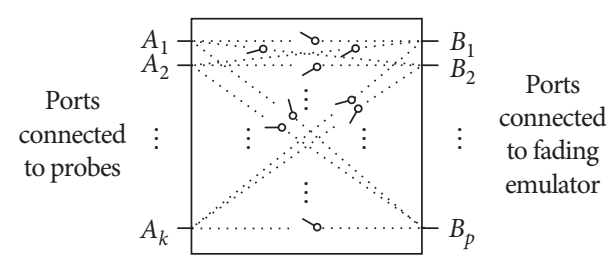

(b)

FIGURE 2: Internal connection structure of the (a) APM network and (b) the switch.

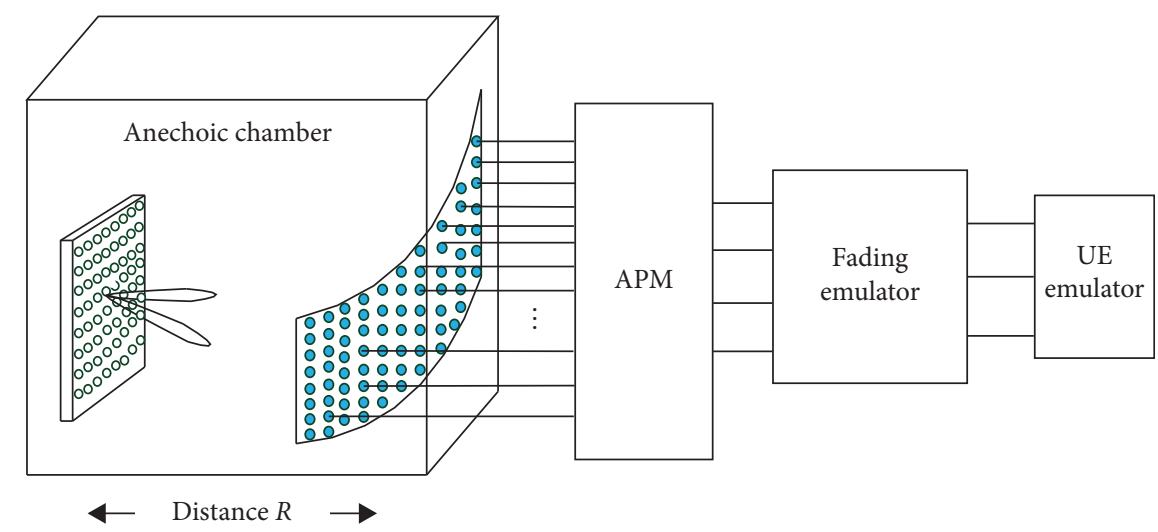

FIgURE 3: MPAC setup with cascaded APM network and CE for the massive MIMO BS.

frequency band signals has simpler structure and lower cost than the channel emulator. The OTA testing system using cascaded APM network and CE in Figure 3 can achieve the effect of multiple channel emulators and control the cost of the testing system. Based on the above descriptions, the APM network will have better performance than the switch system in OTA testing for mmWave massive MIMO devices.

In summary, the APM network can not only overcome the structural defects of the mechanical switch described above but also improve the testing performance of the OTA setup. The outputs of the $\mathrm{CE}$ and the probes used for channel reconstruction are no longer one-to-one mapping, but $P$-to$K$. Adjusting the link parameters of the APM network properly and increasing the number of probes installed on the probe wall appropriately, the reconstructed channel can be obtained accurately. The specific implementation process and performance analysis will be detailed in Section 3. The effectiveness of the proposed MPAC setup for reconstructing the static channel model and dynamic channel model is verified in Section 4.

\section{Implementation Process of the Cascaded MPAC OTA Setup}

In the OTA testing system, spatial correlation can be used to evaluate the reconstructed radio channel [10]. However, there are several major problems using spatial correlation to evaluate reconstructed mmWave channels. Firstly, spatial correlation is used as a statistical measure of the similarity of the received signals, without comparing continuous angular spectrum and emulated angular spectrum synthesized by probes directly. In this process, if the number of sampling points' test area is finite and the specific parameters of the PAS are not considered, such as angular spread (AS) and 
azimuth angle of arrival (AoA), two completely different angular spectrums may result in similar spatial correlation [10]. Secondly, for the sub- $6 \mathrm{GHz}$ OTA testing system, the spatial correlation is used to estimate the reconstruction of the channel directly. However, the mmWave massive MIMO systems are more directive and the channel is highly sparse and specular; therefore, spatial correlation function might be less relevant to determine OTA system performance [6]. Finally, the mmWave massive MIMO antennas have the beamforming capability, which should be studied when testing the performance of the DUT. Therefore, the selected evaluation metrics need to emphasize the performance of the beam, e.g., beam acquisition, tracking, refinement, and recovery [6]. Based on the above description, angular spectrum is selected as the test metric in the sequel.

\subsection{Power Angular Spectrum}

3.1.1. Target Power Angular Spectrum. Suppose that there is a virtual planar antenna array in the test area, which consists of $M$ antenna elements. Using the classical Bartlett beamformer under a given geometry antenna array, the angular spectrum can be obtained by scanning the signal power in each DoA through spatial filtering [11]. Therefore, the target angular spectrum can be calculated for the reference channel model using the following equation [12]:

$$
P(\Psi)=\mathbf{a}^{H}(\Psi)\left[\oint \mathbf{e}(\Phi) P(\Phi) \mathbf{e}^{H}(\Phi) \mathrm{d} \Phi\right] \mathbf{a}(\Psi),
$$

where $\mathbf{a}(\Psi)$ is the normalized steering vector for spatial direction $\Psi$ which consists of the azimuth $\phi$ and the elevation $\theta$. Assuming the ideal far-field conditions, the expression of the steering vector is $\mathbf{a}(\Psi)=[(1 / \sqrt{M}) \exp (j(2 \pi$ $\left./ \lambda)\left(\left(\overrightarrow{o p_{\Psi}} \cdot \overrightarrow{o a_{1}}\right) /\left|\overrightarrow{o p_{\Psi}}\right|\right)\right),(1 / \sqrt{M}) \exp \left(j(2 \pi / \lambda)\left(\left(\overrightarrow{o p_{\Psi}} \cdot \overrightarrow{o a_{2}}\right) /\right.\right.$ $\left.\left.\left.\left|\overrightarrow{o p_{\Psi}}\right|\right)\right), \ldots,(1 / \sqrt{M}) \exp \left(j(2 \pi / \lambda) \quad\left(\left(\overrightarrow{o p_{\Psi}} \cdot \overrightarrow{o a_{M}}\right) /\left|\overrightarrow{o p_{\Psi}}\right|\right)\right)\right]^{T}$, and $\mathbf{e}(\Phi)=\left[e_{1}(\Phi), e_{2}(\Phi), \ldots, e_{M}(\Phi)\right]^{T} \in \mathbb{C}^{M \times 1}$ is a channel response vector with respect to signal direction $\Phi$, where $\{\cdot\}^{T}$ and $\{\cdot\}^{H}$ represent the transpose and conjugate transpose of a matrix or vector, respectively. The DUT is placed in the center of the test area, $\overrightarrow{o p_{\Psi}}$ represents the direction vector of incoming wave direction $\Psi$ to the center origin $o$, and $\overrightarrow{o a_{m}}$ is the vector from the antenna element $m$ of the BS to the center origin $o$, where $m=1,2, \ldots, M$. Covariance matrix $\mathbf{R} \in \mathbb{C}^{M \times M}$ of the received signals of the BS antenna is given by

$$
\mathbf{R}=\oint \mathbf{e}(\Phi) P(\Phi) \mathbf{e}^{H}(\Phi) \mathrm{d} \Phi,
$$

where $P(\Phi)$ is the angular spectrum density function of the angular direction $\Phi$. The spherical angular spectrum needs to satisfy the following normalization condition [13]:

$$
\oint P(\Phi) \mathrm{d} \Phi=\int_{-\pi}^{\pi} \int_{-\pi / 2}^{\pi / 2} P(\theta, \phi) \cos \theta \mathrm{d} \theta \mathrm{d} \phi=1 .
$$

3.1.2. Emulated Power Angular Spectrum. The emulated angular spectrum is synthesized by a limited number of probes in the anechoic chamber, which is affected primarily by the probe positions and the power weights. Suppose that there are $K$ available probes, the angular space set is $\left\{\Phi_{k}\right\}$ and the corresponding probe weight set is $\left\{w_{k}\right\}, k=1,2, \ldots, K$. According to the expression of the target angular spectrum, the emulated angular spectrum is [12] given by

$$
\begin{aligned}
\widehat{P}(\Psi, w) & =\mathbf{a}^{H}(\Psi) \widehat{R} \mathbf{a}(\Psi) \\
& =\mathbf{a}^{H}(\Psi) \sum_{k=1}^{K} \mathbf{e}\left(\Phi_{k}\right) w_{k} \mathbf{e}^{H}\left(\Phi_{k}\right) \mathbf{a}(\Psi),
\end{aligned}
$$

where $w_{k}$ is the allocated power for the $k$ th probe and $K$ is the total number of the probes selected in the OTA experiment.

3.1.3. Objective Function. The target angular spectrum and emulated angular spectrum generated by the OTA probes are given in (1) and (4), respectively. Each vector element of the angular spectrum is a superposition of the radiant power and energy leakage from the DUT to each location on the probe wall. In order to determine the weights of probes to reconstruct the target channel, the optimization problem aiming at minimizing the deviation between the target and the emulated angular spectrum can be given by

$$
\begin{array}{ll}
\min _{\mathbf{w}} & \|P(\Psi)-\widehat{P}(\Psi, \mathbf{w})\|_{2}^{2} \\
\text { s.t. } & \|\mathbf{w}\|_{1}=1, \quad 0 \leq w_{k} \leq 1 .
\end{array}
$$

It is noted that equation (5) is a quadratic programming problem with linear constraints, which can be solved by convex optimization techniques readily.

3.2. Implementation Process. Proper probe selection can accurately reconstruct target channels and control the cost of the OTA testing setup. The multishot algorithm for the probe selection is described in [13]. The idea of the algorithm is to remove the probe with the smallest weight for each iteration and select an optimal subset of the candidate probes until the channel emulator resources $\left(\operatorname{Pr}_{P}\right.$ output ports) are occupied completely. Different from switch, the advantage of the APM network is that $P r_{K}$ probes are used $\left(P r_{K}>P r_{P}\right)$ for each cluster without increasing the channel emulator. However, $\operatorname{Pr}_{P}$ probes are a subset of $\operatorname{Pr}_{K}$ probes for the cluster using multishot algorithm, and the relationship between $\operatorname{Pr}_{K}$ and $\operatorname{Pr}_{P}$ is $\operatorname{Pr}_{P}=\operatorname{Pr}_{K}-\sum_{q=0}^{K-P-1} k_{q}$, where $k_{q}$ is the number of the deleted probes for the $q$ th iteration. In this way, the optimal probes which are required can be selected as much as possible.

Assuming that the APM network is connected to $K$ probes which are selected and the weights $\mathbf{w} \in \mathbb{R}^{K \times 1}$ of the probes are obtained in equation (5) to synthesize the clusters of the dynamic channel in each sampling time. For simplicity and without loss of generality, the implementation process of the radiated test setup for a link is described in Figure 4 . In addition, given that the delay between clusters is on the order of nanoseconds and the response time of APM is on the order of milliseconds, the MPAC testing setup with a cascaded model should reflect power-delay profiles (PDPs) accurately between the clusters. Therefore, the weight of each 
cluster is controlled so that each output port of the channel emulator only outputs a series of impulse responses of a single cluster in Figure 4. Clusters with different delays are synthesized separately. During cluster synthesis, the total sampling time of the channel is assumed to be $T$. The impulse responses of the channel emulator are defined as $\mathbf{G}=\{\mathbf{G}(p, t)\} \in \mathbb{C}^{P \times T}$, and $\mathbf{G}(p, t)$ represents the impulse responses of the $p$ output port at time $t, t=1,2, \ldots, T$ and $p=1,2, \ldots, P$. It should be noted that the number of the clusters $L$ should be less than or equal to the number of the output ports of the channel emulator $P$ (where we assume $L=P$ ). The restriction is proper for the mmWave channel reconstruction in [14], where the number of the identified clusters ranges from 8 to 12 in the non-line-of-sight (NLOS) scenario. Furthermore, each OTA antenna contributing to the cluster has independent fading coefficient sequences with identical statistics [8]. Therefore, the impulse responses which are output by cross-connected APM network need to ensure that probes are independently and identically distributed (i.i.d.). That is, the impulse response G passes the APM network and generates the i.i.d. impulse response $\mathbf{H}=\left\{\mathbf{H}_{t}(k, p)\right\} \in \mathbb{C}^{K \times P \times T}$ which is the expected target channel and mapped to probes through the output ports of the APM network.

Considering $P$ clusters and achieving the exact mapping of the impulse responses of each cluster to the $K$ output ports of the APM network at time $t$, there is a transition matrix $\mathbf{B}_{t} \in \mathbb{C}^{K \times P}$ which satisfies

$$
\underset{\mathbf{B}_{t}, p=1,2, \ldots, P}{\operatorname{minimize}}\left\|\left[\begin{array}{c}
\sqrt{w_{1}} \cdot \mathbf{H}_{t}(1, p) \\
\sqrt{w_{2}} \cdot \mathbf{H}_{t}(2, p) \\
\vdots \\
\sqrt{w_{K}} \cdot \mathbf{H}_{t}(K, p)
\end{array}\right]-\left[\begin{array}{c}
\mathbf{B}_{t}(1, p) \cdot \mathbf{G}(p, t) \\
\mathbf{B}_{t}(2, p) \cdot \mathbf{G}(p, t) \\
\vdots \\
\mathbf{B}_{t}(K, p) \cdot \mathbf{G}(p, t)
\end{array}\right]\right\|
$$

where $\mathbf{B}_{t}$ represents the transition matrix of the APM network at sample time $t$, and the weights $\left\{w_{k}\right\}$ are obtained in (5). The rows of the obtained transition matrix $\mathbf{B}_{t}$ correspond to the output ports of the APM network, and the number of columns is the output ports of the channel emulator. Each element represents the adjusted amplitude and phase of the APM network at sample time $t$. Given the sample time $t=1,2, \ldots, T$, using the i.i.d. impulse responses $\mathbf{H}$ which would be recreated accurately by the cascaded model and impulse responses $\mathbf{G}$, complex weight matrix $\mathbf{B} \in \mathbb{C}^{K \times P \times T}$ would be obtained.

Naturally, through the transition matrix $\mathbf{B}_{t}(p) \in \mathbb{C}^{K \times 1}$ and the impulse response $\mathbf{G}(p, t) \in \mathbb{C}$ generated by the $p$ th port of the channel emulator, the i.i.d. impulse responses $\mathbf{H}_{t}(p) \in \mathbb{C}^{K \times 1}$ of $K$ output ports of the APM network can be synthesized to achieve the effect of increasing probes at sampling time $t$ :

$$
\mathbf{H}_{t}(p)=\mathbf{B}_{t}(p) \cdot \mathbf{G}(p, t)
$$

sampling time $t$ and port of the channel emulator $p$ can be traversed to obtain channel impulse responses $\mathbf{H}$.

3.3. Capacity Evaluation. Channel capacity reflects transmitted rate ability of the channel and is an important measure of the wireless communication, which can be improved effectively by using antenna array technology. Therefore, massive MIMO arrays at the BS can be utilized to improve the transmission rate of the MIMO communications. Besides the previous work focused on angular spectrum accuracy at Rx side, the impact of the Tx side antenna arrays and the propagation environments would be considered as well in this subsection. The reference channel models, i.e., GSCMs, and the channel emulation methods (PFS) are introduced later. Then, we use the MIMO rate capacity [15] as a metric to study the accuracy of the emulated channel using the APM network and the switch in the OTA testing setup, respectively.

Through the uplink established in the antenna systems, the channel capacity can be expressed by the Shannon formula:

$$
C(t)=\frac{1}{N_{f}} \sum_{n_{f}=1}^{N_{f}} \log _{2} \operatorname{det}\left(\mathbf{I}+\frac{\gamma}{N_{t}} \cdot H\left(t, n_{f}\right) \cdot H\left(t, n_{f}\right)^{H}\right)
$$

where $\gamma$ is the signal-to-noise ratio, $N_{f}$ is the number of subcarriers, and $N_{t}$ is the number of transmitter antennas. $\mathbf{H}_{\text {ref }}\left(t, n_{f}\right) \in \mathbb{C}^{U \times S}$ and $\mathbf{H}_{\text {ota }}\left(t, n_{f}\right) \in \mathbb{C}^{U \times S}$ are the reference channel matrix and emulated channel matrix in the frequency domain, respectively, which can be obtained by performing the Fourier transform of the channel impulse responses $\left\{H_{u, s}^{\mathrm{ref}}(t, \tau)\right\}$ and $\left\{H_{u, s}^{\text {ota }}(t, \tau)\right\}$ in Appendix A. Through (7), reference channel capacity $\mathbf{C}_{\text {ref }}(t)$ and emulated channel capacity $\mathbf{C}_{\text {ota }}(t)$ are simulated to analyze the accuracy of the OTA emulated channels which are recreated by the APM network and the switch, respectively.

\section{Simulation Results}

In this section, simulation results are provided to verify the performance of the MPAC setup with a cascaded model. Assume that the massive MIMO DUT operates at $28 \mathrm{GHz}$. The angular spectrum is used as the performance metric, and the simulation experiments are carried out under both static and dynamic channel scenarios. Uniform line array (ULA) on the Tx side consists of 4 antennas which are ideal dipoles, and mutual coupling effect between antennas is ignored. There is an $\mathrm{Rx}$ antenna array which is composed of $8 \times 8$ omnidirectional antennas. In simulations, it is assumed that the distance $R$ from the DUT center to the probe wall is far enough to meet the far-field condition. In addition, the multipath channels are simulated with 10000 time samples and a sampling interval is defined as 1 millisecond. Therefore, the update rate of the APM network in simulations is also defined as 1 millisecond to generate the expected impulse responses $\mathbf{H}$. The power angular spectrums of the target clusters obey the Laplacian distribution which is defined in [15]. Without loss of generality, 91 probes are placed fixedly on the probe wall in $\left[-30^{\circ}, 30^{\circ}\right]$ of the horizontal positions with $5^{\circ}$ intervals and $\left[-15^{\circ}, 15^{\circ}\right]$ of the vertical positions with $5^{\circ}$ intervals, which are used in the cascaded testing model. In addition, the multishot algorithm for the probe selection is used to select optimal 8 probes 


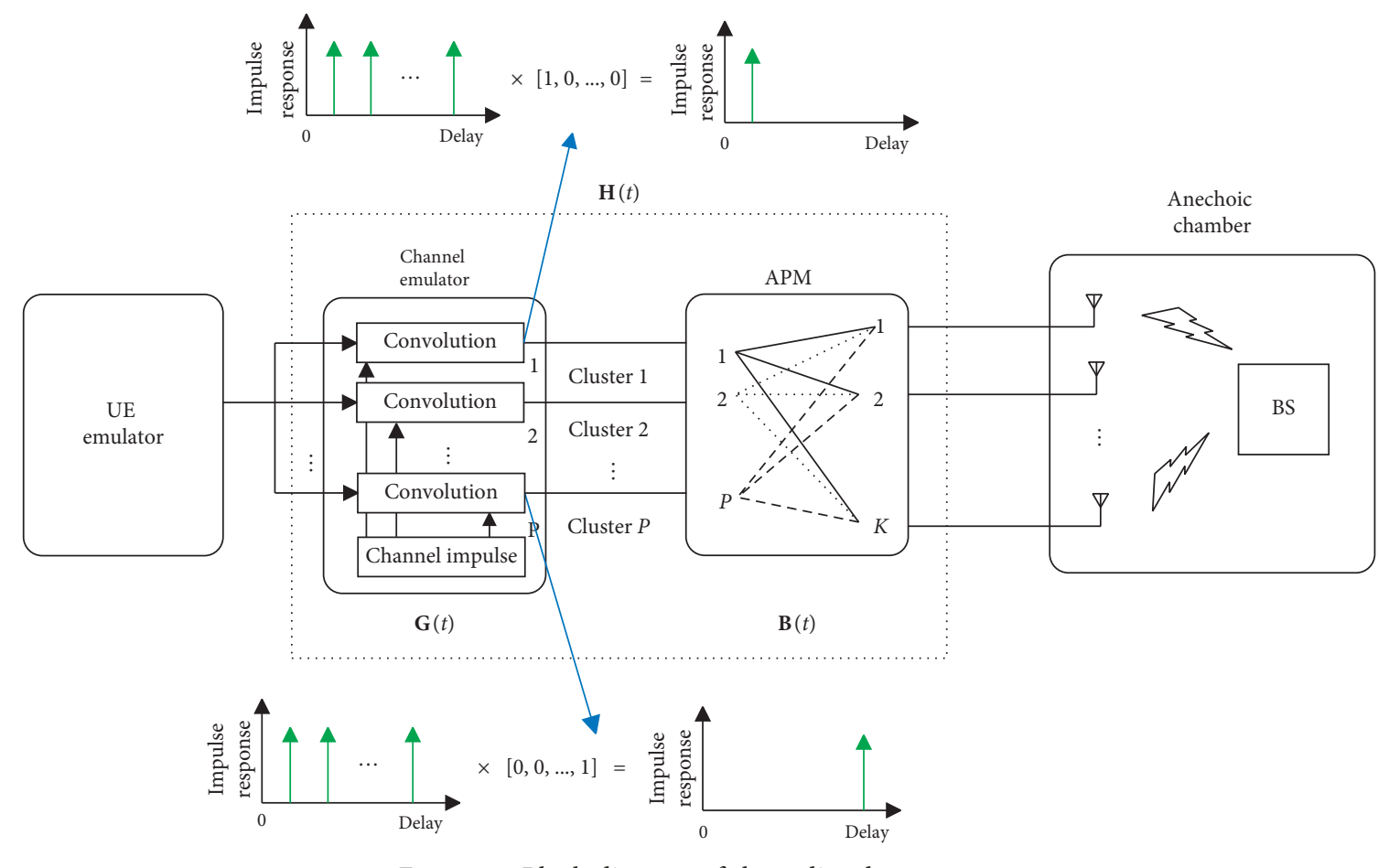

FIGURE 4: Block diagram of the radiated test setup.

from 91 probe antennas in the OTA testing setup with the switch. For simplicity, we will only discuss the situations for single cluster hereafter. In this study, ignoring the uncontrollable effects of the mechanical switch and the APM network, we assume that they are completely free to perform the switching at each considered moment without any distortion, and upper bounds of the performance for OTA testing setups with switch and APM network are given in the simulations, respectively.

4.1. Static Angular Spectrum. The performance of the proposed testing setup is considered for the static channel model. Angular spectrums observed by the DUT through Bartlett beamforming are compared. From equation (1) and equation (4), the target angular spectrum and the emulated angular spectrum can be calculated conveniently. Figure 5 depicts the simulation result of the target angular spectrum of a single cluster which obeys truncated Laplacian distributed 3D PAS with ASA $=10^{\circ}$ and $\mathrm{ESA}=3^{\circ}$ (CDL-B model [16]), and its AoA and EoA are assumed to be $0^{\circ}$ and $0^{\circ}$, respectively. The other structures of the MPAC testing system have not been changed. Figures 6(a) and 6(b) represent emulated angular spectrum emulated by the APM network and the switch, respectively. The peak of the target angular power is $15.13 \mathrm{~dB}$, which has been illustrated in Figure 5. Intuitively, compared with the angular spectrum in Figure 6(b), the emulated angular spectrum using the APM network in Figure 6(a) is more similar to the target angular spectrum. According to the numerical analysis, the peak value deviation between the target and the emulated angular spectrum in Figure 6(a) is insignificant, which is $0.01 \mathrm{~dB}$, indicating that the array gain in the emulated channel is

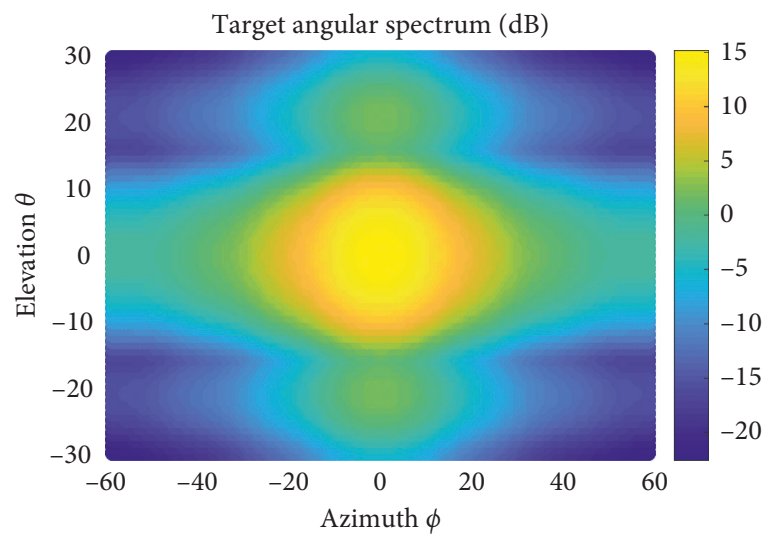

Figure 5: Target angular spectrum with $\mathrm{AoA}=0^{\circ}, \mathrm{Eo} A=0^{\circ}$, $\mathrm{ASA}=10^{\circ}$, and $\mathrm{ESA}=3^{\circ}$.

accurate, and the error of the emulated angular spectrum in Figure 6(b) is $0.14 \mathrm{~dB}$. Obviously, the angular spectrum in Figure 6(a) reconstructs the target angular spectrum in Figure 5 more accurately than that in Figure 6(b).

In Figure 7, using the APM network and the switch, respectively, the errors between the emulated angular spectrum and the target angular spectrum for the direction $\Psi$ are defined as $10 \log P(\Psi)-10 \log \widehat{P}(\Psi, w)$. In Figure 7(a), the image of the error is symmetrical. Since the mean spatial angle of the target PAS is defined as $\left(0^{\circ}, 0^{\circ}\right)$, and the installed probes are symmetrically distributed, the symmetry phenomenon of the Figure 7(a) can be comprehended easily. In addition, the r.m.s. error describes the average variance distance between the target angular spectrum and the emulated angular spectrum, and the r.m.s. errors are $-16.65 \mathrm{~dB}$ and $-4.18 \mathrm{~dB}$ in Figures $7(\mathrm{a})$ and $7(\mathrm{~b})$, respectively. 


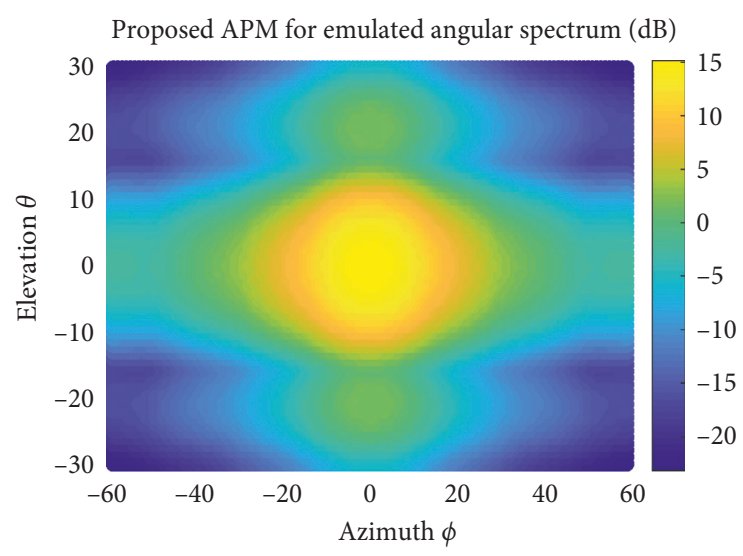

(a)

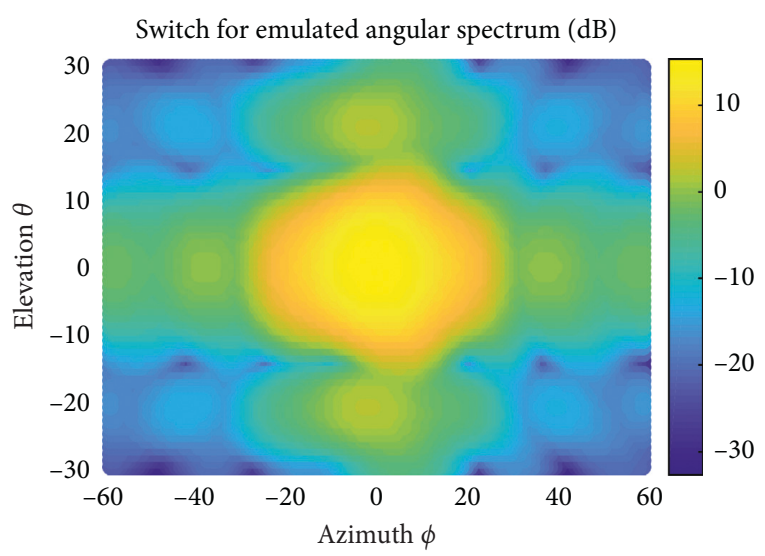

(b)

FIgURE 6: Emulated angular spectrums with the APM network (a) and the switch (b).

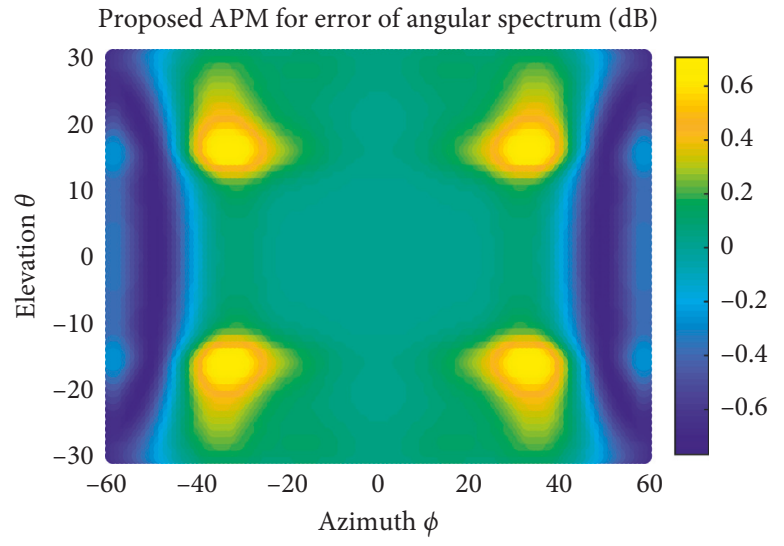

(a)

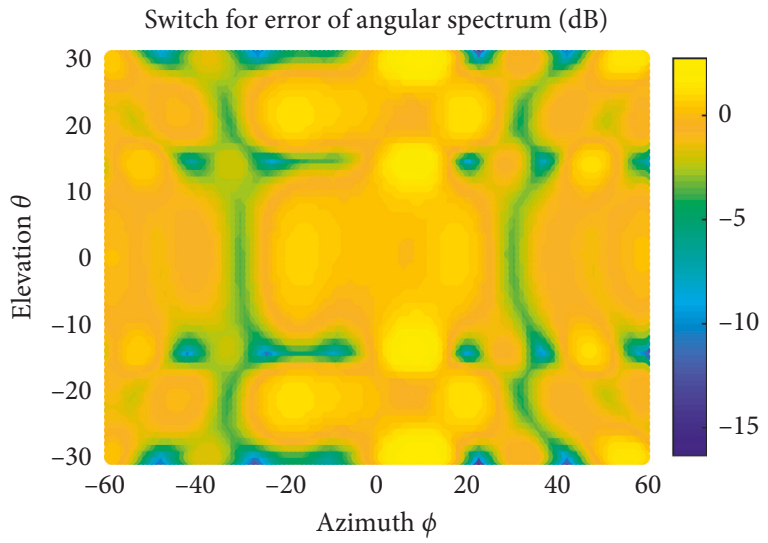

(b)

FIGURE 7: Errors of the angular spectrum with the APM network (a) and the switch (b).

Furthermore, a large number of simulations are performed under different cluster settings to verify the performance of the MPAC testing setup using a cascaded model. Besides CDL-B link model in [16], the typical CDL-A model and CDL-C model described in [16] are simulated, and their results are given in Table 1. As summarized in Table 1, the proposed APM network can reproduce the angular spectrum with higher precision, which is advantageous for verifying the performance of the mmWave massive MIMO BS accurately.

4.2. Dynamic Angular Spectrum. Beamforming is the key technology of the mmWave BS and should be reflected in the reconstructed dynamic channel of the OTA testing. Therefore, compared the APM network with the switch, the emulated dynamic angular spectrums are discussed in the sequel. The angular spectrum can emphasize the performance of the beam, e.g., beam acquisition, tracking, refinement, and recovery. To reflect the dynamic channel intuitively, the angular spectrum is described by adding a time dimension. Without loss of generality, referencing CDL-B in [16], the ASA is $10^{\circ}$ and ESA is defined as $3^{\circ}$. The azimuth target angle is increased from $-10^{\circ}$ to $10^{\circ}$ with $0.1^{\circ}$ intervals, and the elevation angle is decreased from $10^{\circ}$ to $-10^{\circ}$ with $0.1^{\circ}$ intervals gradually. Therefore, there are 201 time samples. Figure 8 describes the dynamic target angular spectrum, where each slice represents an angular spectrum at a different time samples.

It is difficult for the visual presentation to analysis $4 \mathrm{D}$ simulation pictures, and therefore, the target azimuth angular spectrum and the elevation with time samples are simulated in Figures 9(a) and 9(b), respectively. From the angular spectrum in Figure 9, azimuth AoA and elevation EoA change from $-10^{\circ}$ to $10^{\circ}$ and $10^{\circ}$ to $-10^{\circ}$, respectively, which can describe the variety of the spatial direction $\Psi$. Due to the angle spread of the cluster and limited resolution of the Bartlett beamformer, there are power spreads for the variety of the dynamic channel, which have been presented at the target angular spectrum.

To achieve performance comparison between the APM network and the switch, a proper simulation scenario needs to be set up. Depending on the distribution of clusters at any sampling time, the probes are arranged evenly at intervals of $5^{\circ}$ in the horizontal direction $-30^{\circ}$ to $30^{\circ}$ and vertical direction $-15^{\circ}$ to $15^{\circ}$ of the probe wall, which can be used by 
TAвLE 1: Emulation deviations for different cluster settings.

\begin{tabular}{|c|c|c|c|c|c|c|c|}
\hline \multicolumn{4}{|c|}{ Cluster settings } & \multicolumn{2}{|c|}{ Angular spectrum with APM } & \multicolumn{2}{|c|}{ Angular spectrum with switch } \\
\hline $\operatorname{AoA}\left({ }^{\circ}\right)$ & $\operatorname{EoA}\left({ }^{\circ}\right)$ & $\operatorname{ASA}\left({ }^{\circ}\right)$ & $\operatorname{ESA}\left({ }^{\circ}\right)$ & |peak| (dB) & r.m.s (dB) & |peak| (dB) & r.m.s (dB) \\
\hline-20 & 10 & 10 & 3 & 0.0031 & -5.2557 & 0.0438 & -3.7885 \\
\hline-20 & 10 & 5 & 3 & 0.00017 & -15.2405 & 0.00034 & -9.1467 \\
\hline-20 & 10 & 2 & 3 & 0.0017 & -17.1357 & 0.002 & -14.2699 \\
\hline 5 & -5 & 10 & 3 & 0.0030 & -14.5862 & 0.0585 & -3.3436 \\
\hline 5 & -5 & 5 & 3 & 0.00039 & -26.9030 & 0.0069 & -6.1667 \\
\hline 5 & -5 & 2 & 3 & 0.00085 & -22.3349 & 0.0019 & -14.0239 \\
\hline 10 & -5 & 10 & 3 & 0.0027 & -11.5519 & 0.1405 & -3.9020 \\
\hline 10 & -5 & 5 & 3 & 0.00043 & -26.5943 & 0.00071 & -8.9643 \\
\hline 10 & -5 & 2 & 3 & 0.00082 & -22.5181 & 0.0019 & -14.0001 \\
\hline
\end{tabular}

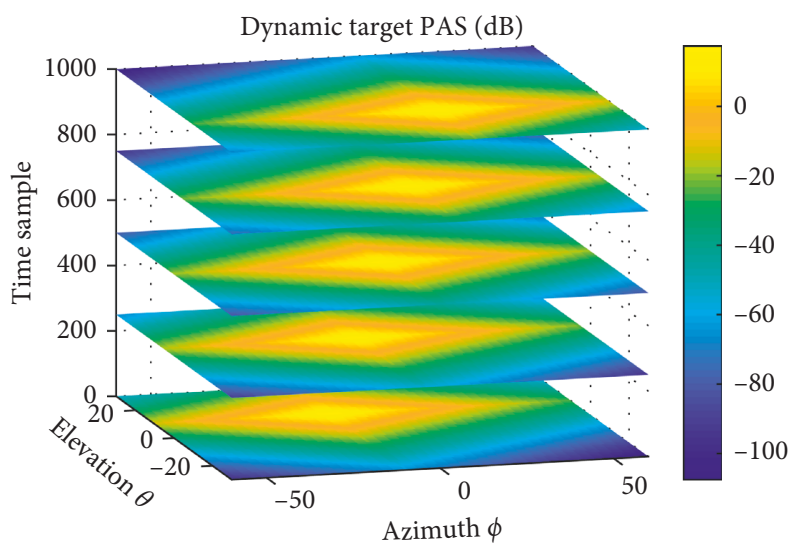

Figure 8: Dynamic target PAS of the cluster with time samples. ASA $=10^{\circ}, \mathrm{ESA}=3^{\circ}$, AoA ranges from $-10^{\circ}$ to $10^{\circ}$, and EoA ranges from $-10^{\circ}$ to $10^{\circ}$.

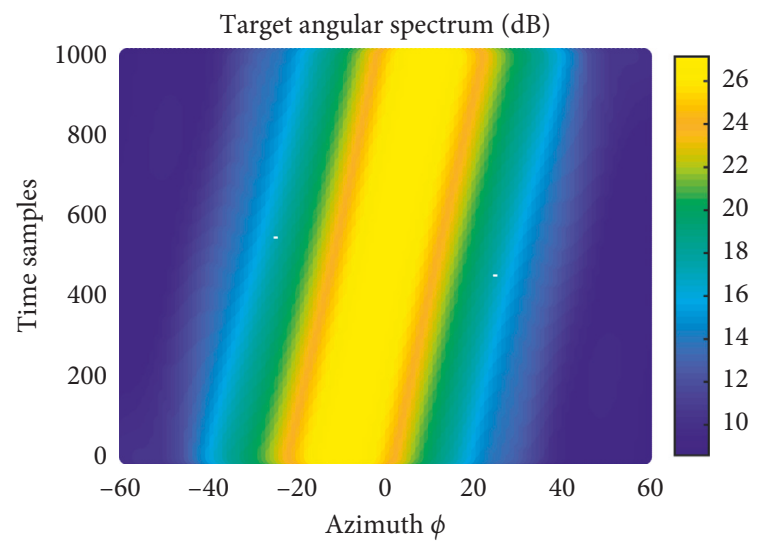

(a)

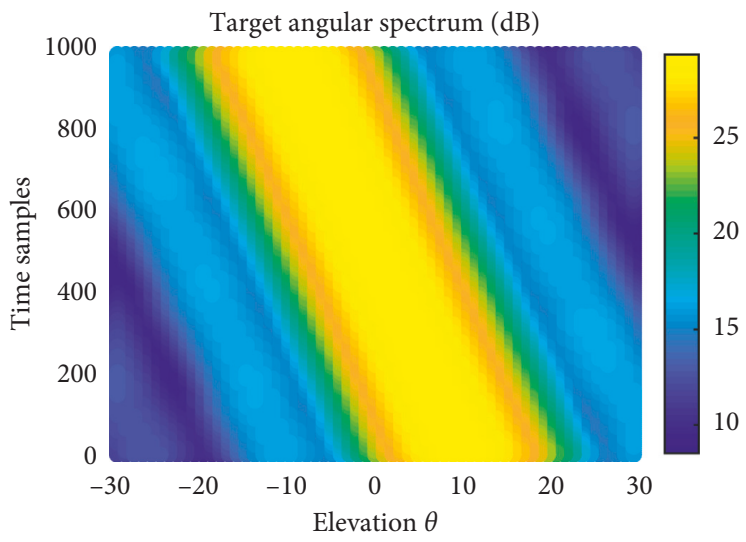

(b)

Figure 9: Power distribution of target azimuth and elevation in the dynamic channel model. AoA ranges from $-10^{\circ}$ to $10^{\circ}$, EoA ranges from $-10^{\circ}$ to $10^{\circ}, \mathrm{ASA}=10^{\circ}$, and $\mathrm{ESA}=3^{\circ}$.

the cluster fully at all sampling moments. Using the fully connected APM network system, all of the above 91 probes can be used to reconstruct the target channel. In addition, given the limited resources of the channel emulators, it is assumed that 8 probes are selected for the channel reconstruction using the multishot selection strategy. The scenario represents an existing switch configuration system.
According to equation (5), the emulated angular spectrums under two different systems are obtained. Figure 10 illustrates the emulated azimuth and elevation angular spectrum for the dynamic channel model with the APM network. The simulations use all 91 probes at each time. The vertical axis of the two subgraphs (Figures 10(a) and 10(b)) represents time samples, and the horizontal axes represent the azimuth $\psi$ and elevation $\theta$, respectively. It can be seen 


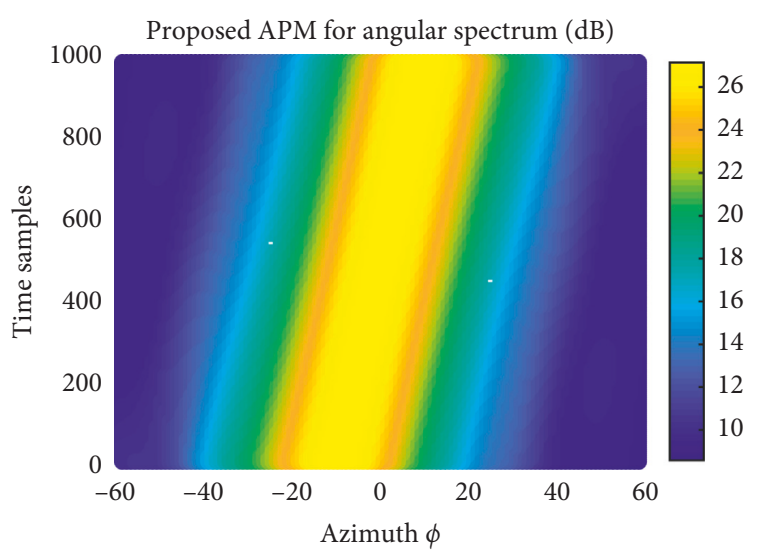

(a)

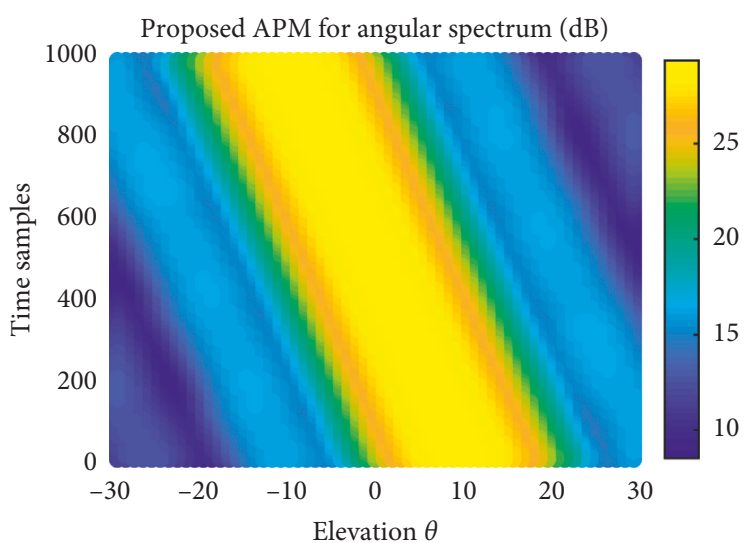

(b)

FIGURE 10: APM network for azimuth and elevation angular spectrum for a dynamic channel model.

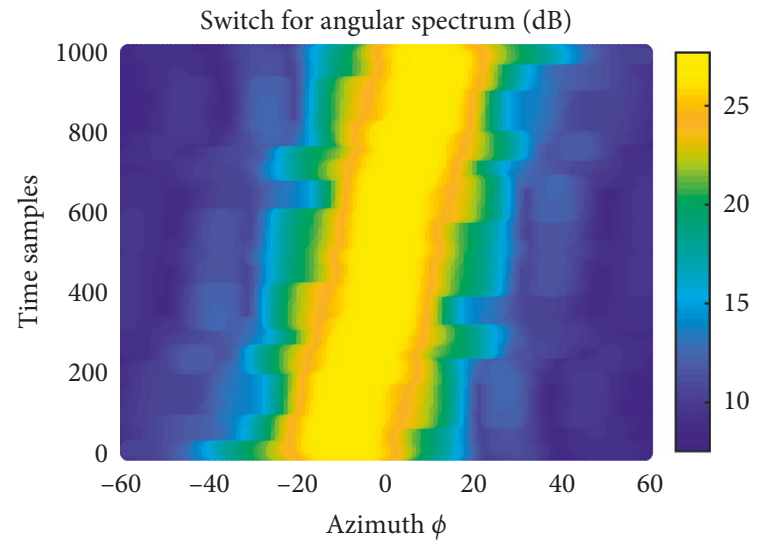

(a)

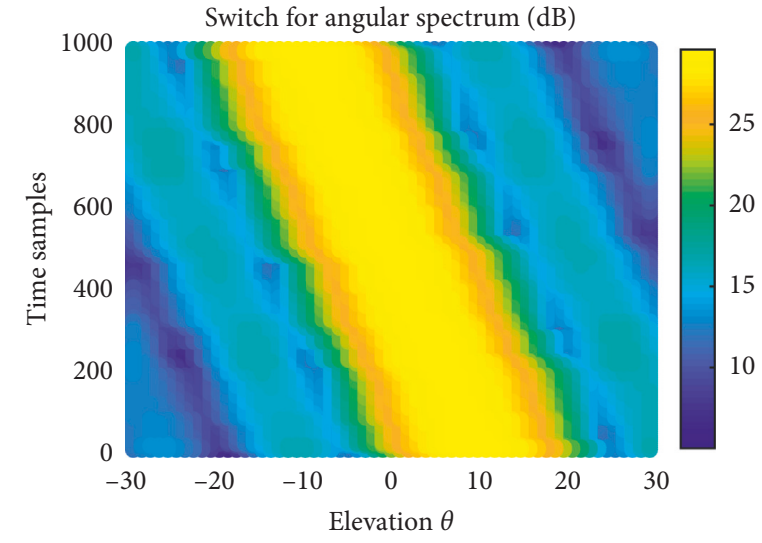

(b)

FIGURE 11: Switch system for azimuth and elevation angular spectrum for a dynamic channel model.

that the subgraphs in Figure 10 can match very well with the target angular spectrum. Compared with Figure 9, the angular spectrums of Figure 11 are obviously different. Although the characteristics of the beam acquisition and tracking can be realized, the beam power is significantly disturbed by other directions. The reconstructed channel has significant errors compared with the MPAC setup using a cascaded model.

Next, the angular spectrums in Figures 9-11 are compared numerically. Figure 12 describes the peak powers of the target angular spectrum and the emulated angular spectrum using the APM network and the switch at different times. As above, the peak powers are given from the azimuth and elevation at different sampling times, and the existing differences can be observed clearly. It is clear that the peak powers emulated by the APM system coincide with the target peak powers completely at different times in Figure 12. However, for the existing switch system, the deviations of the peak powers are obvious. In addition, in order to show the overall differences between the target angular spectrum and emulated angular spectrum, Figure 13 presents the r.m.s. errors obtained at different sampling times. From the obtained r.m.s. errors, the

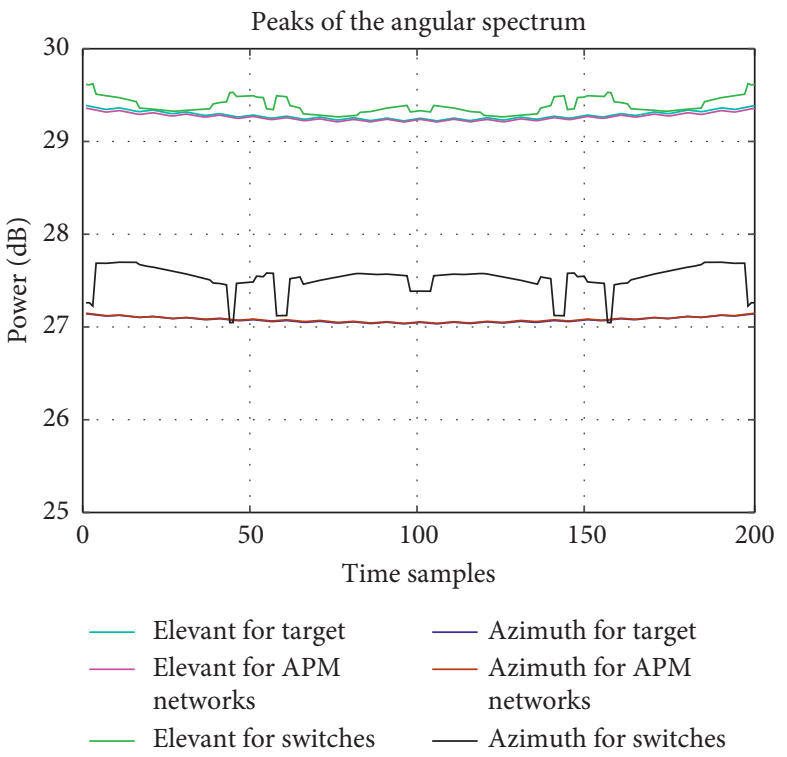

FIGURE 12: The peak powers of the angular spectrum at sampling time. 


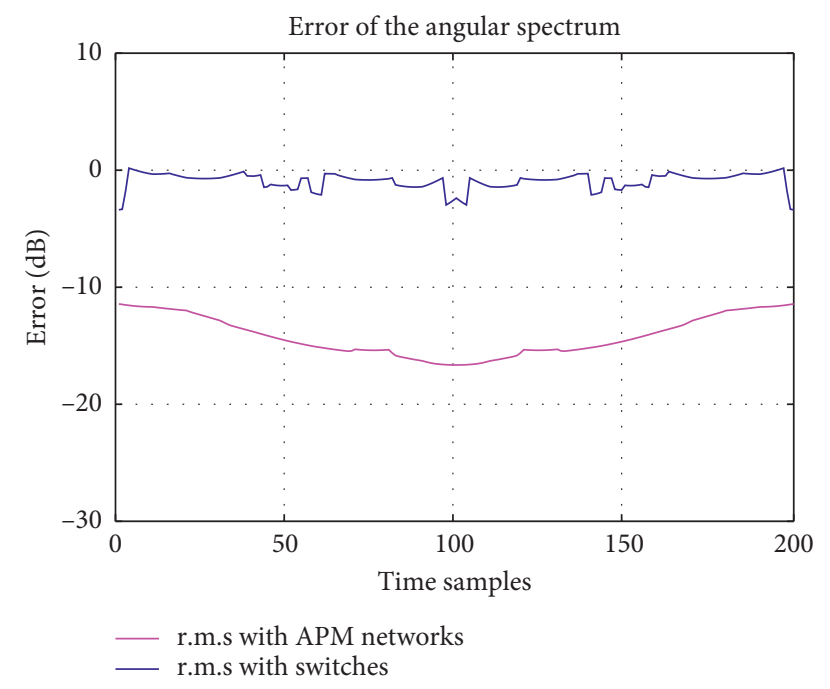

FIgURE 13: The r.m.s. errors of the angular spectrum at sampling times.

mean deviation of the angular spectrum simulated by the APM network is lower $10 \mathrm{~dB}$ than the angular spectrum emulated by the switch at each sampling time. It is verified that the reference channel can be reconstructed more accurately by increasing the number of probes without changing the size of the test area.

Another point to note is that all curves show symmetrical characteristics, which is reasonable due to the following reasons. Firstly, the DUT antennas in the test area are symmetric with respect to the center of the DUT panel in simulation. Secondly, the probes which are placed on the probe wall are distributed evenly, and the probe positions are symmetrical on the probe wall. Thirdly, at different sampling moments, the mean spatial angle of the cluster is symmetrical with respect to the center of the probe wall (AoA ranges from $-10^{\circ}$ to $10^{\circ}$ and EoA ranges from $10^{\circ}$ to $-10^{\circ}$ ). Based on these three reasons, the symmetry presented in the simulation diagram can be explained.

4.3. Channel and the Rate Capacity. Under ideal far-field conditions, the signal-to-noise ratio is $\gamma=20 \mathrm{~dB}$ and the power of the target cluster is 1 . The channel coefficients consist of the $64 \times 4$ CIR matrix from the UE antennas to the DUT elements. $\mathbf{F}_{\text {OTA }}$ is ideal polarimetric antenna pattern vector $[0,1]$ of the OTA probes, and the polarimetric antenna pattern vector $\mathbf{F}_{s, \mathrm{UE}}$ is defined as $[1,0]^{T}$ for the UE. The elements of the polarization matrix are $\alpha_{l, q}^{V V}=$ $\exp \left(j \Phi_{l, q}^{V V}\right), \quad \alpha_{l, q}^{H V}=\sqrt{\kappa_{l, q}} \exp \left(j \Phi_{l, q}^{H V}\right), \quad \alpha_{l, q}^{V H}=\sqrt{\kappa_{l, q}} \exp$ $\left(j \Phi_{l, q}^{V H}\right)$, and $\alpha_{l, q}^{H H}=\exp \left(j \Phi_{l, q}^{H H}\right) . \Phi_{l, q}^{V V}, \Phi_{l, q}^{H V}, \Phi_{l, q}^{V H}$, and $\Phi_{l, q}^{H H}$ are i.i.d. random variables which are uniformly distributed over $[0,2 \pi] . \kappa_{l, q}$ is the XPR of the qth subpath of the $l$ th cluster.

Figures 14 and 15 present instantaneous channels and capacities under 10000 time samples for the reference and emulated SCME CDL-B channel model. Instantaneous amplitudes of the reference channel, the estimated channel with APM network, and the estimated channel with switch are simulated in Figure 14, respectively. It can be seen from the instantaneous channels of different receiving antennas that the average power is close to 1 within the sampling time. As expected, comparing $\mathbf{H}_{5}(t, \tau)$ with $\mathbf{H}_{6}(t, \tau)$ or comparing $\mathbf{H}_{60}(t, \tau)$ with $\mathbf{H}_{61}(t, \tau)$, the channel amplitude variations of the receiving antennas at adjacent locations are approximately same. The first-order statistical feature of the reference channel and the emulated channel is approximately consistent. In Figure 15, the vertical axis of the figure represents the transmission rate per unit time of unit bandwidth. In order to compare the APM network with the switch system visually, the average channel capacity is presented. Through numerical calculation, the average channel capacity of the reference channel for single receiver antenna is about 10.02 bits/s/Hz, and the emulated capacity with APM network and switch is $9.98 \mathrm{bits} / \mathrm{s} / \mathrm{Hz}$ and $9.92 \mathrm{bits} / \mathrm{s} / \mathrm{Hz}$, respectively.

Due to the fact that three instantaneous curves are messy, we plot the cumulative distribution functions (CDFs) of the capacity results for scenario CDL-B shown in Figure 16, switch and reference channel. Compared with the emulated channel generated by the switch, the CDF of the emulated channel generated by the APM network matches well with the reference channel model. Thus, based on the simulation results of both channel capacity and angular spectrum, the MPAC testing system with the APM network can reconstruct the radio channel environment better than the switch system.

The simulation results show that the cascaded model in this paper is effective. However, some practical problems may be considered in the future testing process. On the one hand, accuracy errors of the amplitude modulation and phase modulation may have impact on recreated channel, which are less than $1.4^{\circ}$ and $0.5 \mathrm{~dB}$, respectively. On the other hand, digital APM network may also have some uncertain hardware problems, i.e., insertion loss and isolation. In addition, given the sampling rate of the channel emulator and APM network, it may be difficult to create a high-speed channel environment. As a future work, we will further study the influence of the above nonideal factors on channel reconstruction. 


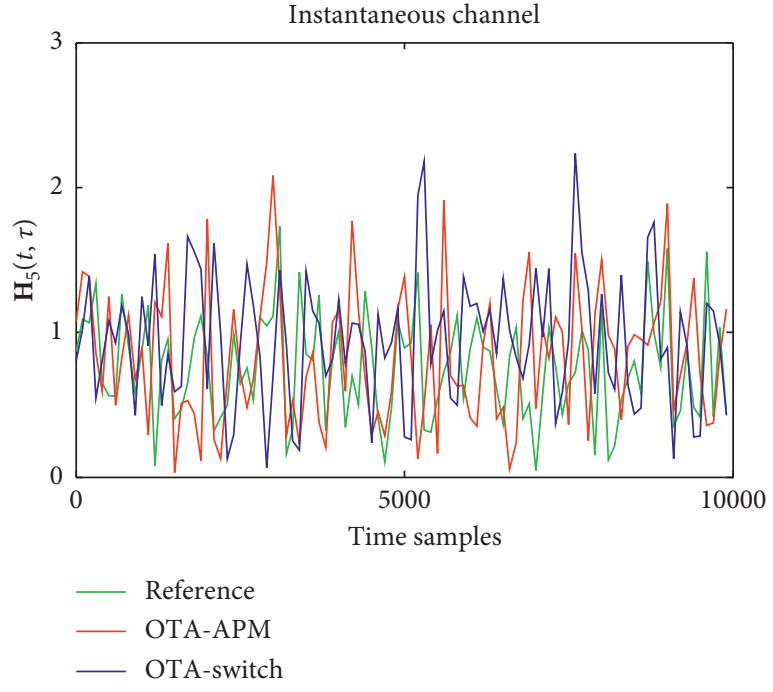

(a)

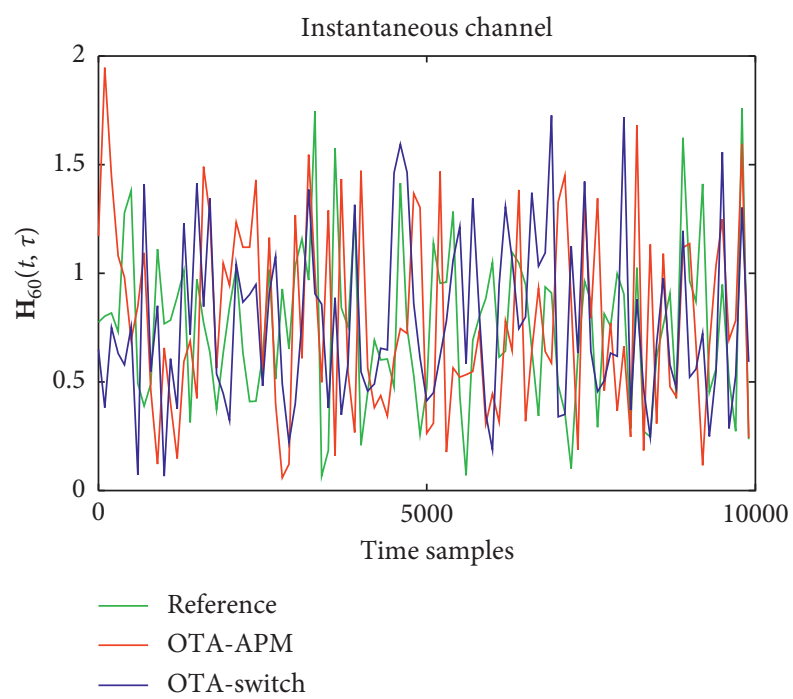

(c)

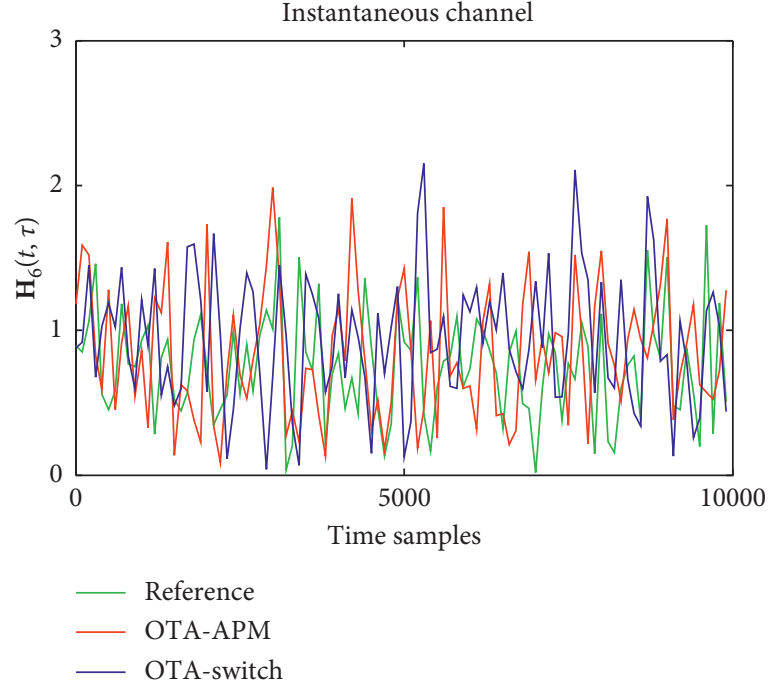

(b)

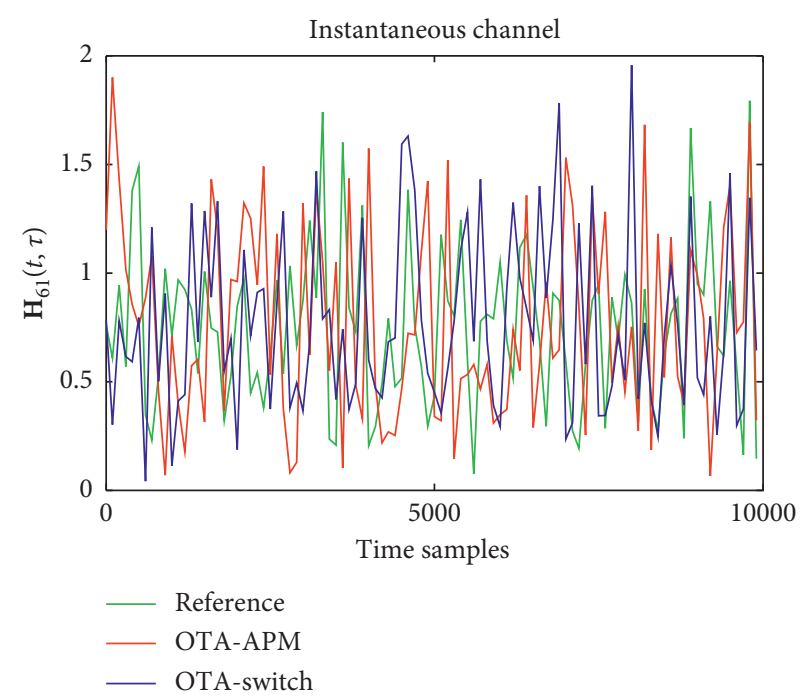

(d)

FIGURE 14: Instantaneous channels of the receiver antennas 5, 6, 60, and 61 for the reference and emulated SCME CDL-B channel model, $\mathbf{H}_{5}(t, \tau), \mathbf{H}_{6}(t, \tau), \mathbf{H}_{60}(t, \tau)$, and $\mathbf{H}_{61}(t, \tau)$.

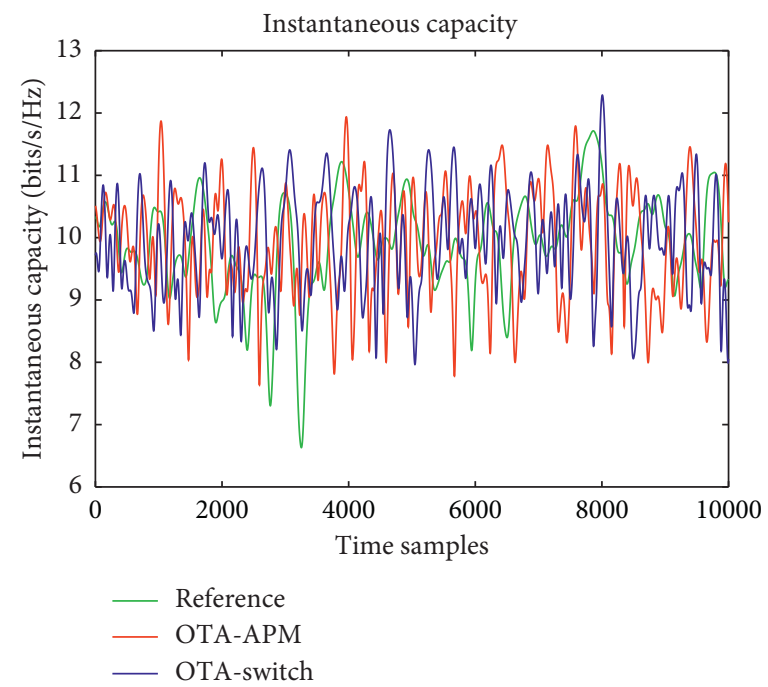

Figure 15: Instantaneous capacities for the reference and emulated SCME CDL-B channel model. The SNR $\sigma$ is $20 \mathrm{~dB}$. 


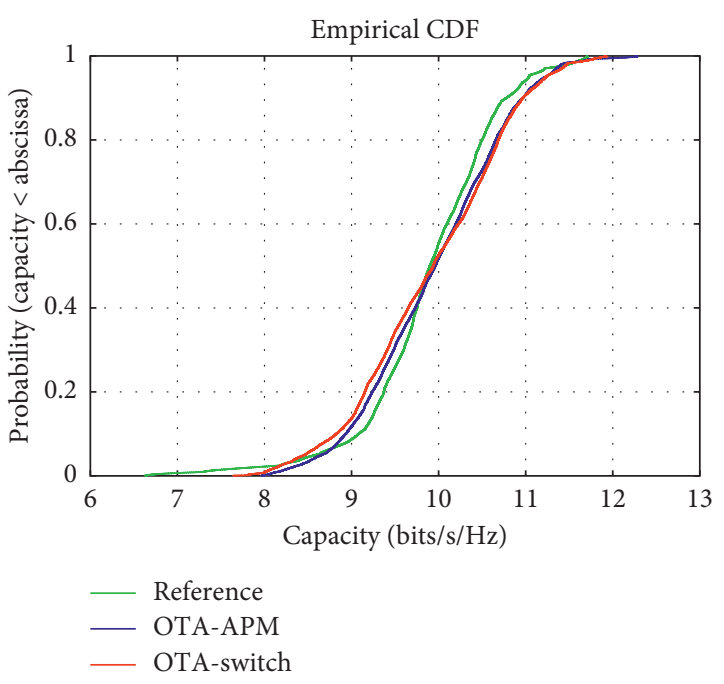

FIGURE 16: CDFs of the channel capacity for scenario CDL-B with average SNR $\sigma=20 \mathrm{~dB}$.

\section{Conclusion}

We have proposed a MPAC testing setup for mmWave massive MIMO devices using cascaded APM network and CE. The internal structure of the APM network and the drawbacks of mechanical switch for the mmWave devices testing are introduced in detail. The paper uses spherical angular spectrum to determine probe weights instead of using the spatial correlation. Considering the resolution of the BS antenna array and the shortcomings of the spatial correlation itself, the angular spectrums are simulated to measure the reconstructed mmWave channel accurately.

Further, simulations for the single cluster based on the static and dynamic channel models have been done in this paper, confirming the superiority of the proposed APM network. In static channel model, the cluster is simulated by the angular spectrum, and the peak power and r.m.s. error are compared using the APM network and switch, respectively. For testing the mmWave massive MIMO devices, dynamic channel model and test environment have been considered carefully. Reconstructing a time-variant dynamic channel model, the weights must be updated frequently according to the channel changes. The time dimension is added to show the accuracy of the angular spectrum estimated under dynamic channel conditions. In addition, the channel capacity as a performance measure is also studied. All simulations give encouraging results for testing the performances of massive MIMO devices using MPAC setup with APM network. In addition, what needs to be made clear is that the probe configuration for multiclusters should be considered in real environment. Given that multiclusters will share the selected probes, the performance of the proposed OTA testing setup will be more prominent than for single cluster.

\section{Appendix}

\section{A}

A.1. Reference Channel Models. The ray-based channel model is a GSCM which is selected in the study at the same frequency. One simplicity of the GSCM is its ability to separate into the propagation environments, antenna at the BS and user equipment side. For the reference model, the channel impulse response (CIR) matrix $\mathbf{H}_{\text {ref }}(t, \tau) \in \mathbb{C}^{U \times S}$ can be generated in [17]. For a MIMO system that deploys $U$ antenna elements at the BS side and $S$ antenna elements at the UE side, the MIMO channel $\mathbf{H}_{\text {ref }}(t, \tau)$ can be represented by

$$
\mathbf{H}_{\mathrm{ref}}(t, \tau)=\left[\begin{array}{ccc}
h_{1,1}(t, \tau) & \cdots & h_{1, S}(t, \tau) \\
\vdots & \ddots & \vdots \\
h_{U, 1}(t, \tau) & \cdots & h_{U, S}(t, \tau)
\end{array}\right],
$$

where the time-variant radio channel transfer function $h_{u, s}(t, \tau)$ can be written as

$$
h_{u, s}(t, \tau)=\sum_{l=1}^{L} h_{u, s, l}(t, \tau)
$$

where $L$ is the number of clusters and $t$ is the time. For each receiver and transmitter element pair $(u, s)$, the channel coefficient for $l$ th cluster can be expressed as [17]

$$
\begin{aligned}
& h_{u, s, l}(t, \tau)=\sqrt{\frac{P_{l}}{Q}} \sum_{q=1}^{Q}\left[\begin{array}{c}
F_{s, U E}^{V}\left(\phi_{l, q}\right) \\
F_{s, U E}^{H}\left(\phi_{l, q}\right)
\end{array}\right]^{T}\left[\begin{array}{cc}
\alpha_{l, q}^{V V} & \alpha_{l, q}^{V H} \\
\alpha_{l, q}^{H V} & \alpha_{l, q}^{H H}
\end{array}\right] \cdot\left[\begin{array}{c}
F_{u, B S}^{V}\left(\varphi_{l, q}\right) \\
F_{u, B S}^{H}\left(\varphi_{l, q}\right)
\end{array}\right] \\
& \cdot \exp \left(j 2 \pi v_{l, q} t\right) \cdot \exp \left(j 2 \pi \vec{r}_{t x, l, q} \cdot \mathbf{d}_{t x, s}\right) \\
& \cdot \exp \left(j 2 \pi \vec{r}_{r x, l, q} \cdot \mathbf{d}_{r x, u}\right) \cdot \delta\left(\tau-\tau_{l}\right),
\end{aligned}
$$

where $\phi_{l, q}, \varphi_{l, q}$, and $v_{l, q}$ are the angle of departure, angle of arrival, and Doppler frequency of the $q$ th subpath of the $l$ th cluster, respectively. $P_{l}$ and $\tau_{l}$ denote the power and delay of the $l$ th cluster. $F_{s, U E}^{V}$ and $F_{s, U E}^{H}$ are the field patterns of UE antenna element $s$ for the vertical and horizontal polarization, respectively. Similarly, $F_{u, B S}^{V}$ and $F_{u, B S}^{H}$ are the field patterns of BS antenna element $u$ for the vertical and horizontal polarization, respectively. Coefficient $\alpha_{l, q}^{a b}$ is the complex amplitude of the $q$ th subpath of the $l$ th cluster for transmit polarization $a$ and receive polarization $b . \overrightarrow{\mathbf{r}}_{r x, l, q}$ is the spherical unit vector with azimuth arrival angle $\phi_{n, m, \mathrm{AOA}}$ and elevation arrival angle $\theta_{n, m, \mathrm{ZOA}} \cdot \vec{r}_{t x, l, q}$ is the spherical unit vector with azimuth departure angle $\phi_{n, m, \mathrm{AOD}}$ and elevation departure angle $\theta_{n, m, Z O D} \cdot \mathbf{d}_{r x, u}$ is the location vector of receiver antenna element $u$ and $\mathbf{d}_{t x, s}$ is the location vector of transmitter antenna element $s$.

A.2. Emulated Channel Models. The radio channels from the UE to the DUT (BS) array are calculated for the OTA testing. For an MPAC setup equipped with $K$ OTA probes, the channel matrix $\mathbf{H}_{\text {ota }}(t, \tau) \in \mathbb{C}^{U \times S}$ is composed of the transfer matrix $\mathbf{V}=\left\{v_{m, k}\right\} \in \mathbb{C}^{M \times K}$ from $K$ probes to $M$ DUT antennas and the fading components of the channel model $\mathbf{H}_{K, S}^{\text {ota }}(t, \tau)=\left\{\sum_{l=1}^{L} h_{k, s, l}^{\text {ota }}(t, \tau)\right\}$ and defined as 


$$
\begin{aligned}
& h_{k, s, l}^{\text {ota }}(t, \tau)=\sqrt{\frac{P_{l}}{Q}} \sum_{q=1}^{Q} \mathbf{F}_{s, \mathrm{UE}}\left(\phi_{l, q}\right)\left[\begin{array}{cc}
\alpha_{l, q}^{V V} & \alpha_{l, q}^{V H} \\
\alpha_{l, q}^{H V} & \alpha_{l, q}^{H H}
\end{array}\right] \mathbf{F}_{\mathrm{OTA}} \\
& \cdot \sqrt{w_{l, k}} \exp \left(j 2 \pi v_{l, q} t\right) \cdot \exp \left(j 2 \pi \vec{r}_{t x, l, q} \cdot \mathbf{d}_{t x, s}\right) \\
& \cdot \delta\left(\tau-\tau_{l}\right), \\
& \mathbf{H}_{\text {ota }}(t, \tau)=\mathbf{V} \cdot \mathbf{H}_{K, S}^{\text {ota }}(t, \tau),
\end{aligned}
$$

where $w_{l, k}$ are the weights of $k$ probe for the lth cluster and $\mathbf{F}_{\text {OTA }}$ is an ideal polarimetric antenna pattern matrix of the OTA probes. In testing, the transmit power of each OTA probe is calibrated to the same level as the calibrated antenna at the center of the test area, and the pathloss caused by the free space propagation from the OTA probe to the test area is omitted.

\section{Data Availability}

The authors claim that all data generated or analysed in this article are provided by the simulations and are included in this published article. The MATLAB data used to support the findings of this study are available from the corresponding author upon request.

\section{Conflicts of Interest}

The authors declare that they have no conflicts of interest.

\section{Acknowledgments}

This work was supported in part by the National Natural Science Foundation of China (Grant no. 61971067), in part by the State Major Science and Technology Special Projects (Grant nos. 2018ZX03001028-003), and in part by the Beijing Municipal Science and Technology Project (Grant no. Z191100007519002). The work of L. Xin was supported by the BUPT Excellent PhD Students Foundation (Grant no. CX2018103).

\section{References}

[1] J. G. Andrews, T. Bai, M. N. Kulkarni, A. Alkhateeb, A. K. Gupta, and R. W. Heath, "Modeling and analyzing millimeter wave cellular systems," IEEE Transactions on Communications, vol. 65, no. 1, pp. 403-430, 2016.

[2] E. G. Larsson, O. Edfors, F. Tufvesson, and T. L. Marzetta, "Massive MIMO for next generation wireless systems," IEEE Communications Magazine, vol. 52, no. 2, pp. 186-195, 2014.

[3] W. A. T. Kotterman, C. Schirmer, M. H. Landmann, and G. Del Galdo, "New challenges in over-the-air testing," in Proceedings of the 2017 11th European Conference on Antennas and Propagation (EUCAP), pp. 3676-3678, Paris, France, March 2017.

[4] P. Kyösti, L. Hentilä, W. Fan, J. Lehtomäki, and M. Latva-Aho, "On radiated performance evaluation of massive MIMO devices in multi-probe anechoic chamber OTA setups," IEEE
Transactions on Antennas and Propagation, vol. 66, no. 10, pp. 5485-5497, 2018.

[5] P. Kyösti, W. Fan, G. F. Pedersen, and M. Latva-Aho, "On dimensions of OTA setups for massive MIMO base stations radiated testing," IEEE Access, vol. 4, pp. 5971-5981, 2016.

[6] W. Fan, P. Kyosti, M. Rumney, X. Chen, and G. F. Pedersen, "Over-the-air radiated testing of millimeter-wave beamsteerable devices in a cost-effective measurement setup," IEEE Communications Magazine, vol. 56, no. 7, pp. 64-71, 2018.

[7] R. E. Mihailovich, M. Kim, J. B. Hacker et al., "MEM relay for reconfigurable RF circuits," IEEE Microwave and Wireless Components Letters, vol. 11, no. 2, pp. 53-55, February 2001.

[8] P. Kyösti, T. Jams, and J.-P. Nuutinen, "Channel modelling for multiprobe over-the-air MIMO testing," International Journal of Antennas and Propagation, vol. 2012, Article ID 615954, 11 pages, 2012.

[9] A. Khatun, V.-M. Kolmonen, V. Hovinen et al., "Experimental verification of a plane-wave field synthesis technique for MIMO OTA antenna testing," IEEE Transactions on Antennas and Propagation, vol. 64, no. 7, pp. 3141-3150, 2016.

[10] W. Fan, X. C. B. Lisbona, F. Sun, J. Ø. Nielsen, M. B. Knudsen, and G. F. Pedersen, "Emulating spatial characteristics of MIMO channels for OTA testing," IEEE Transactions on Antennas and Propagation, vol. 61, no. 8, pp. 4306-4314, 2013.

[11] P. Stoica and R. L. Moses, Spectral Analysis of Signals, Prentice-Hall, Upper Saddle River, NJ, USA, 2005.

[12] Y. Li, L. Xin, and X. Zhang, "On probe weighting for massive MIMO OTA testing based on angular spectrum similarity," IEEE Antennas and Wireless Propagation Letters, vol. 18, no. 7, pp. 1497-1501, 2019.

[13] W. Fan, F. Sun, J. O. Nielsen et al., "Probe selection in multiprobe OTA setups," IEEE Transactions on Antennas and Propagation, vol. 62, no. 4, pp. 2109-2120, 2014.

[14] C. Gustafson, K. Haneda, S. Wyne, and F. Tufvesson, "On mm-Wave multipath clustering and channel modeling," IEEE Transactions on Antennas and Propagation, vol. 62, no. 3, pp. 1445-1455, 2014.

[15] F. Kaltenberger, D. Gesbert, R. Knopp, and M. Kountouris, "Correlation and capacity of measured multi-user MIMO channels," in Proceedings of the2008 IEEE 19th International Symposium on Personal, Indoor and Mobile Radio Communications, pp. 1-5, Cannes, France, September 2008.

[16] ETSI, "Study on channel model for frequencies from 0.5 to 100 GHz," 3GPP 38.901 Tech. Rep. V14.1.1, ETSI, Sophia Antipolis, France, 2017.

[17] W. Fan, P. Kyosti, S. Fan et al., "3D channel model emulation in a MIMO OTA setup," in Proceedings of the 2013 IEEE 78th Vehicular Technology Conference (VTC Fall), pp. 1-5, Las Vegas, NV, USA, September 2013. 


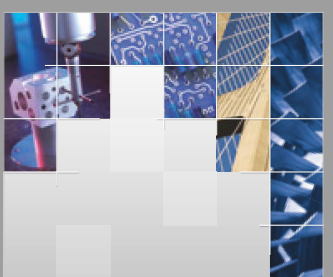

\section{Enfincering}
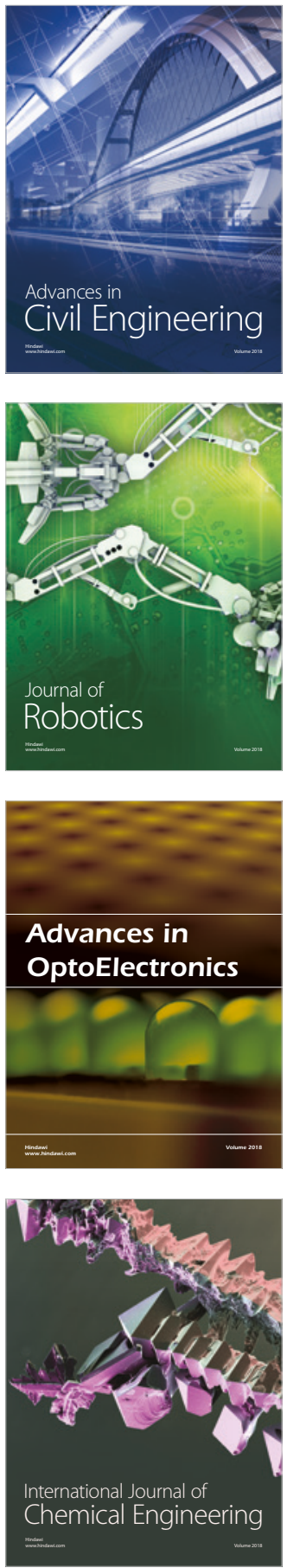

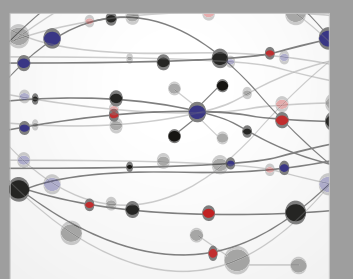

\section{Rotating \\ Machinery}

The Scientific World Journal

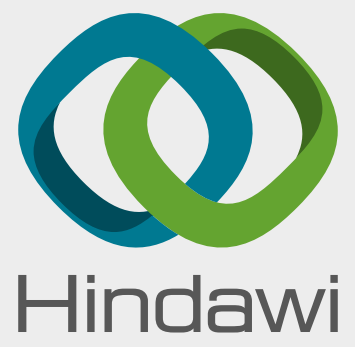

Submit your manuscripts at

www.hindawi.com
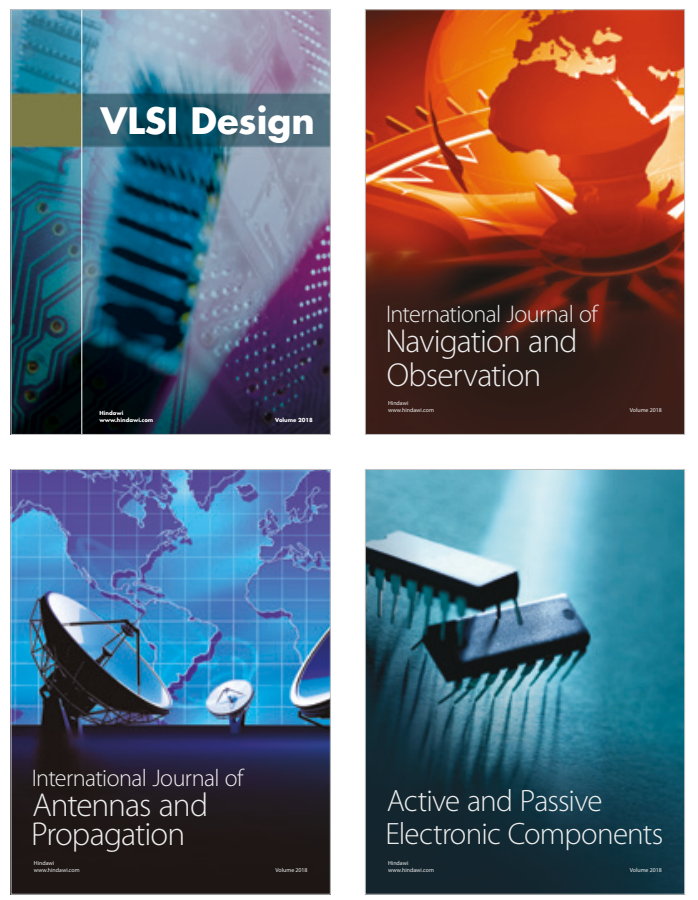
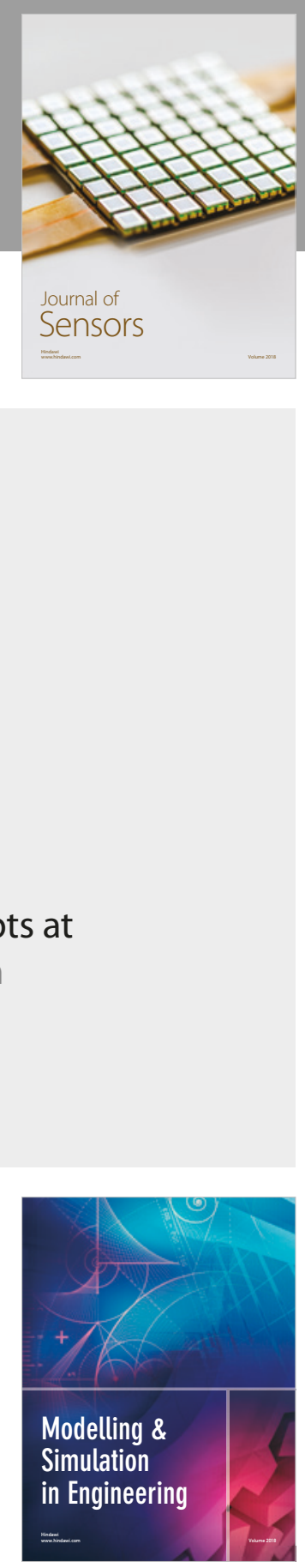

\section{Advances \\ Multimedia}
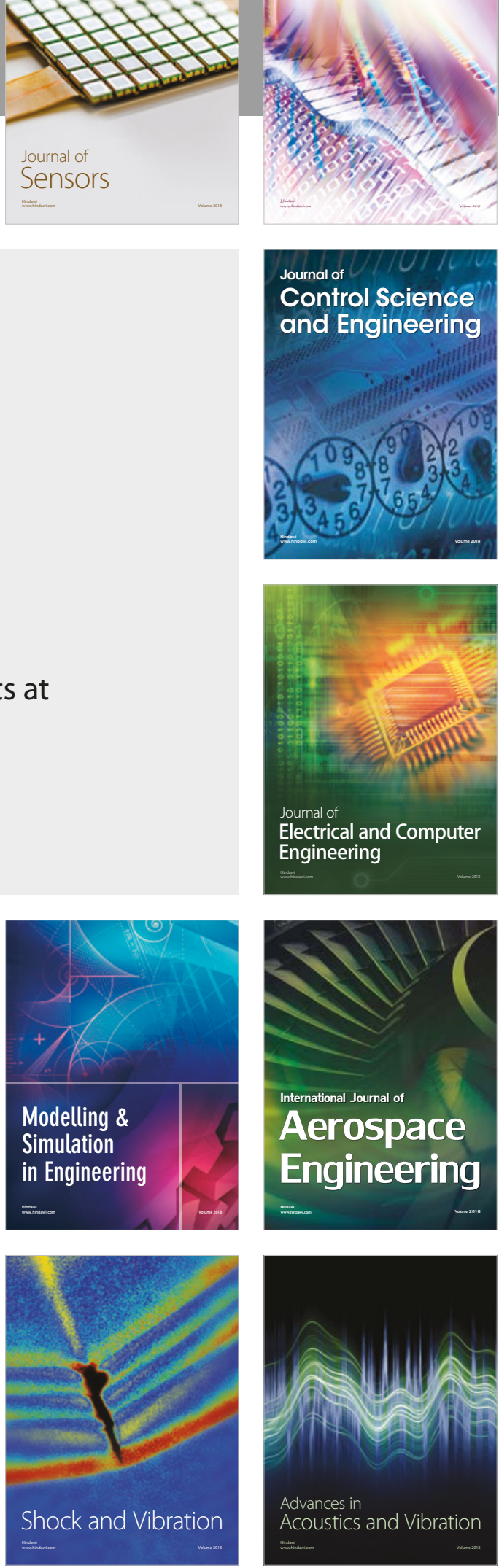